\title{
Core Outcomes and Common Data Elements in Chronic Subdural Hematoma: A Systematic Review of the Literature Focusing on Reported Outcomes
}

\author{
Aswin Chari, Katie C. Hocking,, Ellie Broughton,, ${ }^{1,2}$ Carole Turner,, ${ }^{1,3}$ Thomas Santarius, \\ Peter J. Hutchinson, ${ }^{1,3}$ and Angelos G. Kolias ${ }^{1,3}$
}

\begin{abstract}
The plethora of studies in chronic subdural hematoma (CSDH) has not resulted in the development of an evidence-based treatment strategy, largely due to heterogeneous outcome measures that preclude cross-study comparisons and guideline development. This study aimed to identify and quantify the heterogeneity of outcome measures reported in the CSDH literature and to build a case for the development of a consensus-based core outcome set. This systematic review adhered to the Preferred Reporting Items for Systematic Reviews and Meta-Analyses (PRISMA) statement and was registered with the PROSPERO international prospective register of systematic reviews (CRD42014007266). All full-text English language studies with $>10$ patients (prospective) or $>100$ patients (retrospective) published after 1990 examining clinical outcomes in CSDH were eligible for inclusion. One hundred two eligible studies were found. There were 14 (13.7\%) randomized controlled trials, one single arm trial (1.0\%), 25 (24.5\%) cohort comparison studies, and $62(60.8 \%)$ prospective or retrospective cohort studies. Outcome domains reported by the studies included mortality (63.8\% of included studies), recurrence $(94.1 \%)$, complications $(48.0 \%)$, functional outcomes $(40.2 \%)$, and radiological (38.2\%) outcomes. There was significant heterogeneity in the definitions of the outcome measures, as evidenced by the seven different definitions of the term "recurrence," with no definition given in 19 studies. The time-points of assessment for all the outcome domains varied greatly from inpatient/hospital discharge to 18 months. This study establishes and quantifies the heterogeneity of outcome measure reporting in CSDH and builds the case for the development of a robust consensus-based core outcome set for future studies to adhere to as part of the Core Outcomes and Common Data Elements in CSDH (CODE-CSDH) project.
\end{abstract}

Key words: chronic subdural hematoma; core outcomes; outcome measures

\section{Introduction}

$\mathbf{C}$ Hronic subdural hematoma (CSDH) is one of the most common neurosurgical conditions, with an incidence of approximately $10 / 100,000 /$ year. ${ }^{1-4}$ It is predominantly a disease of increasing age and its incidence is expected to rise, primarily due to a continually aging population and the increasing use of anticoagulant/ antiplatelet medication, both well-recognized risk factors. ${ }^{1-4}$

Surgical treatment of symptomatic CSDH is associated with good neurological recovery in the majority of patients, but recurrent disease still affects $10 \%$ to $20 \%$ of the population, with a resultant impact on functional outcomes and quality of life. ${ }^{3,5}$ Recently, it has been noted that CSDH in the elderly is associated with significant long-term morbidity and mortality and is an indicator of systemic dysfunction analogous to hip fractures. ${ }^{6}$ Therefore, a multi-disciplinary evidence-based approach needs to be adopted for the treatment of this common condition. ${ }^{2,3}$

Despite a plethora of studies investigating the management of $\mathrm{CSDH}$, there remain a number of unanswered questions about the choice of surgical technique, adjuvant therapies, and post-

\footnotetext{
${ }^{1}$ Department of Clinical Neurosciences, Addenbrooke's Hospital and University of Cambridge, Cambridge, United Kingdom.

${ }^{2}$ South West Neurosurgical Centre, Derriford Hospital, Plymouth, United Kingdom.

${ }^{3}$ Surgery Theme, Cambridge Clinical Trials Unit, Cambridge University Hospitals NHS Foundation Trust, Cambridge, United Kingdom.
}

(c) Aswin Chari, et al., 2015; Published by Mary Ann Liebert, Inc. This Open Access article is distributed under the terms of the Creative Commons Attribution Noncommercial License (http://creativecommons.org/licenses/by-nc/4.0/) which permits any noncommercial use, distribution, and reproduction in any medium, provided the original author(s) and the source are credited. 


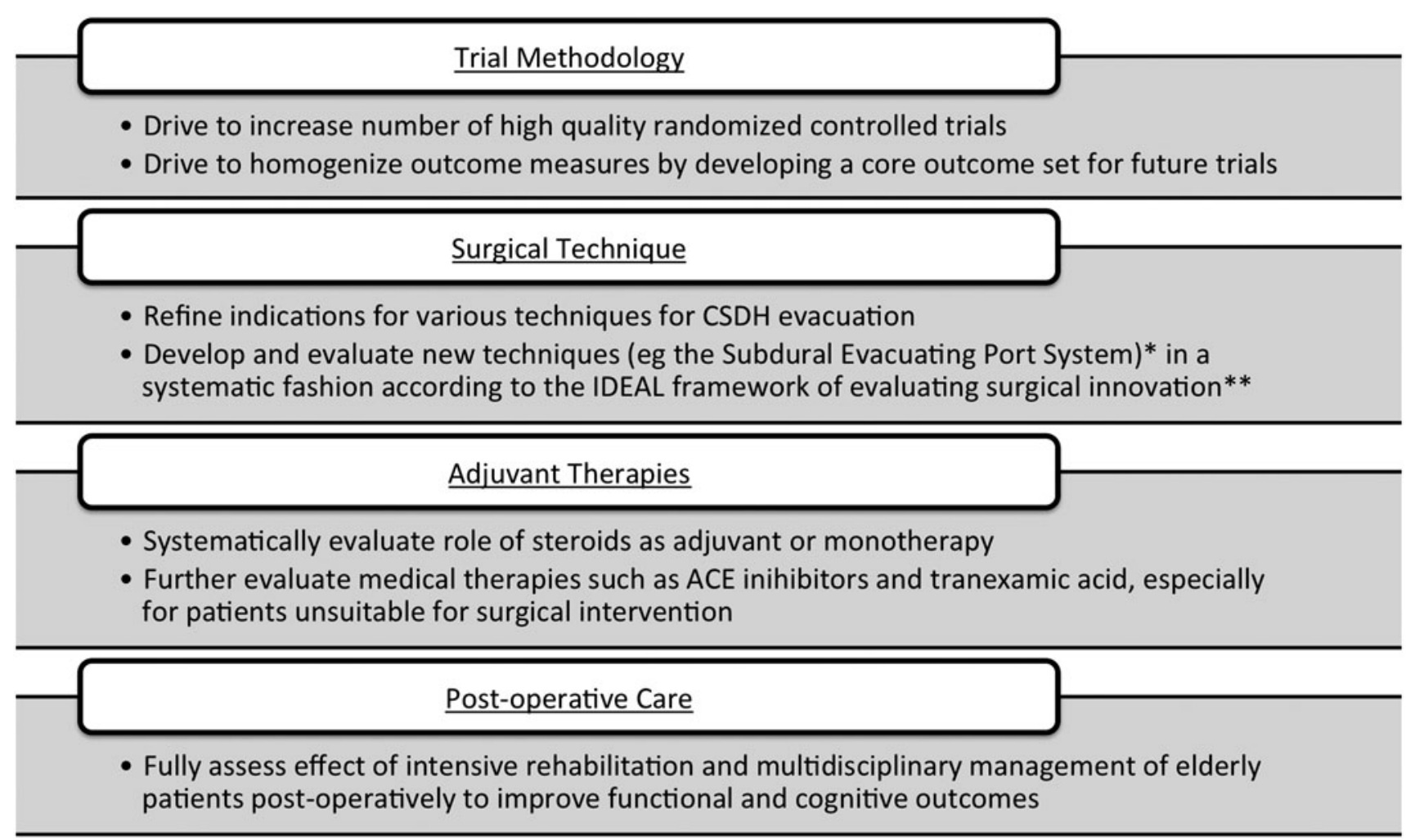

*: Chari A, Kolias AG, Santarius T, et al. Twist-drill craniostomy with hollow screws for evacuation of chronic subdural hematoma. J Neurosurg. 2014 Jul; 121(1):176-83. doi: 10.3171/2014.4.JNS131212.

**: McCulloch P, Altman DG, Campbell WB, et al. No surgical innovation without evaluation: the IDEAL recommendations. Lancet. 2009 Sep 26;374(9695): 1105-12. doi: 10.1016/S0140-6736(09)61116-8.

FIG. 1. Key future research areas surrounding the treatment of CSDH (adapted from Kolias and colleagues). ${ }^{3}$

operative care (Fig. 1). ${ }^{3}$ Many of the studies published in the literature are small retrospective case series reporting disparate baseline data, using variable terminology and definitions of operative technique, and evaluating heterogeneous outcome measures. This poses significant barriers to establishing an evidence-based approach to the management of $\mathrm{CSDH}$, something that has been acknowledged in all three large meta-analyses that have sought to elucidate the optimal treatment options for $\mathrm{CSDH}^{2,4,7}$

To overcome these barriers, clinical trial methodologists have pioneered the development of core outcome sets, "an agreed standardized set of outcomes that should be measured and reported, as a minimum, in all clinical studies and trials in specific areas of health or health care." 8,9 Core outcome sets are currently in use in rheumatoid arthritis, stroke, and traumatic brain injury, and can improve the quality of studies and maximize the potential to combine study outcomes for systematic reviews, meta-analyses, and guideline development. ${ }^{10-12}$ Importantly, the patient-centered methodology used to develop these core outcome sets ensures that outcomes that are important to patients are measured routinely. Funding bodies like the National Institute for Health Research in the UK now recommend the use of core outcome sets, if available, in grant applications for clinical trials. ${ }^{13}$

Developing a core outcome set is a two-stage process. The first step involves reviewing the literature to identify the range of outcome measures used for the particular condition and quantify the heterogeneity that exists in the published literature. The second step involves deriving the core outcome set using a structured consensus process involving all relevant stakeholders, including clinicians, academics, allied health care professionals, patients and carers. The development process for core outcome sets has been facilitated by organizations such as the Core Outcome Measures in Effectiveness Trials initiative. ${ }^{9,14}$

This systematic review represents the first step in the process of developing a core outcome set for CSDH. It aims to identify the range of outcome measures used in the CSDH literature and quantify the heterogeneity, building a case for the development of a consensus-based core outcome set.

\section{Methods}

This systematic review adhered to the Preferred Reporting Items for Systematic Reviews and Meta-Analyses (PRISMA) statement and has been registered with the PROSPERO international prospective register of systematic reviews (CRD42014007266). The MEDLINE and EMBASE databases were searched for the terms "chronic AND subdural" on January 10, 2014. Titles and abstracts were screened for relevance. Full-text articles were then assessed for eligibility according to the following inclusion criteria: English language full text; publication date post-1990; examining clinical outcomes of adult patients with $\mathrm{CSDH}$; and prospective study with $>10$ patients or retrospective study with $>100$ patients.

The reference lists of eligible studies and relevant review articles were scanned for further studies not identified by the search strategy. The search strategy is summarized in Figure 2. Full text manuscripts were requested from corresponding authors of studies that were not accessible.

Relevant data from included studies was collected independently by two authors $(\mathrm{AC}, \mathrm{KCH})$ via a piloted data collection tool. Any discrepancies were settled by consultation between 


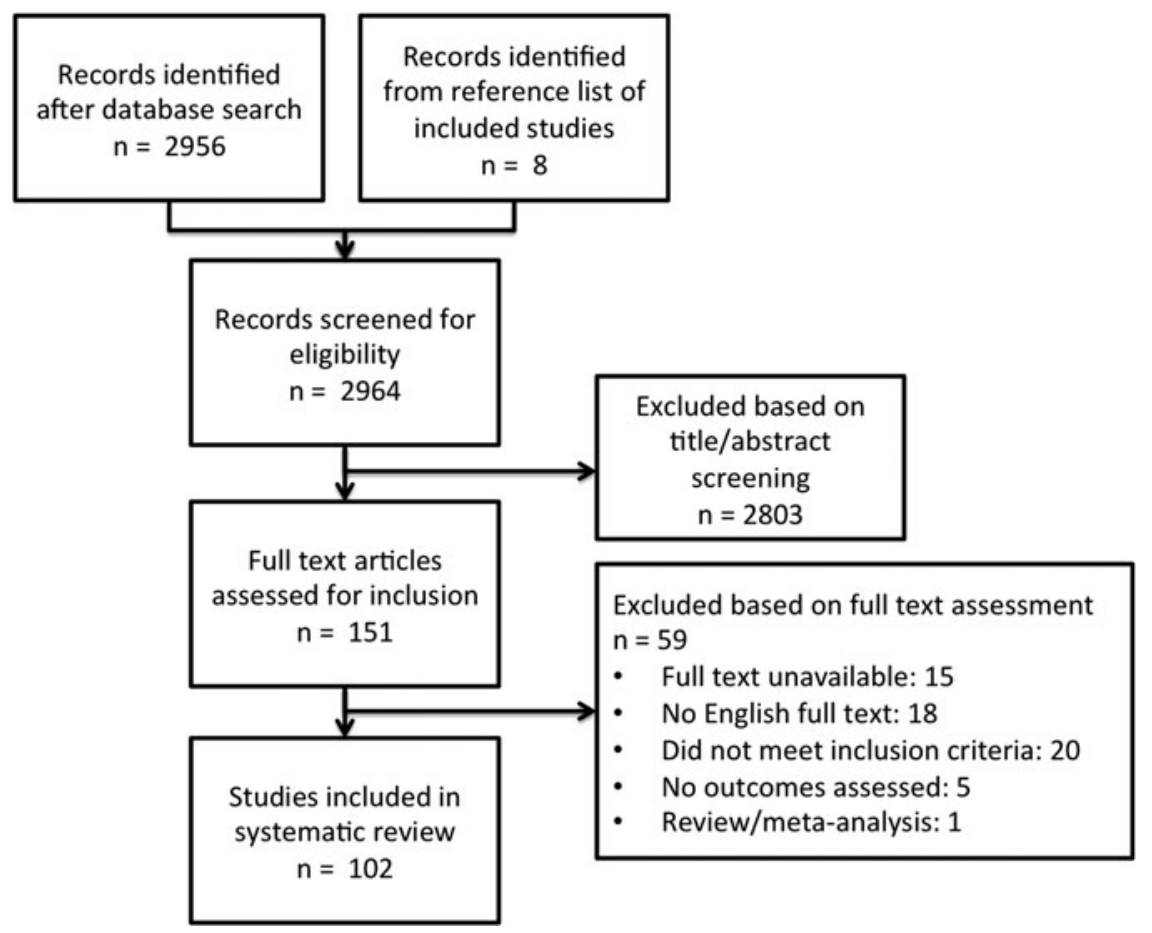

FIG. 2. Search strategy.

the two authors with reference to the original article. Included studies were examined for pre-determined primary and secondary outcomes. All reported outcomes were examined for definitions and time-points. Data was analyzed using Microsoft Excel ${ }^{\circledR}$ (Microsoft Inc., Redmond, WA).

\section{Results}

\section{Study details}

A total of 102 studies were eligible for inclusion, comprising 21,598 patients. Thirty-two (31.4\%) were prospective studies, 66 $(64.8 \%)$ were retrospective and four $(3.9 \%)$ had a combination of prospective and retrospective data. There were $14(13.7 \%)$ randomized controlled trials, one (1.0\%) single arm trial, $25(24.5 \%)$ cohort comparison studies, and $62(60.8 \%)$ cohort studies. Over time, the number of CSDH studies seems to be rising, reflecting the increasing incidence and interest in the condition, but the number of prospective studies does not seem to increase by the same proportion (Fig. 3). A risk-of-bias tool was not applied to the included studies as the inherent nature of the present study was to assess risk of bias based on study design and outcome reporting. The complete list of included studies is provided online (supplementary Appendix 1; see online supplementary material at www.liebertpub.com).

\section{Outcomes domains}

A number of different outcome domains were reported by the 102 studies including mortality, recurrence, complications, functional outcomes, and radiological outcomes. Thirty-two (31.4\%) studies had clearly defined primary outcomes; only seven of these had defined primary and secondary outcomes.

\section{Mortality}

Sixty-five studies $(63.8 \%)$ reported patient mortality, of which $15(14.7 \%)$ did not report a time-point for the mortality figure.
In the studies that reported mortality, there was a wide range of time-points, from "post-operative" or "discharge" up to 18 months, with some studies reporting multiple time-points (Fig. 4). Apart from the immediate post-operative period (reported by 25 studies [24.5\%]), the most popular time-point was at 6 months (reported by 10 studies [9.8\%]). One study reported mortality via a Kaplan-Meier curve, with actual follow-up ranging from 4 months to 14 years.

\section{Recurrence}

Recurrence was reported by 96 studies (94.1\%), highlighting the importance of this outcome measure in CSDH. Time-points were highly varied with $47(46.1 \%)$ reporting no time-point and the rest ranging between inpatient and 12 months. The most common timepoint was at 3 months (19 studies, 18.6\%; Fig. 5). The definitions of recurrence also were highly variable but generally consisted of a combination of either radiological recurrence, recurrence of symptoms, and/or the need for re-operation ( $n=96$; Table 1). Two studies included reoperations on the contralateral (unaffected) side as recurrences. Interestingly, this heterogeneity persisted in the subgroup of studies looking specifically for recurrence $(n=22$; Table 1$)$ and the higher quality subgroup of randomized controlled trials $(n=12$, Table 1). The most common definition in all the subgroups was "symptomatic and radiological recurrence requiring reoperation."

\section{Complications}

Reporting of complications occurred in 49 (48.0\%) studies, although the completeness of reporting was highly variable. Three (2.9\%) did not have any definition for complications, eight $(7.8 \%)$ reported only a single complication or complications associated with the study intervention (e.g., of steroids or of tissue plasminogen activator, $17(16.7 \%)$ reported only surgical/cranial complications, one (1.0\%) reported only medical complications, and $20(19.6 \%)$ reported a comprehensive list of medical and surgical 


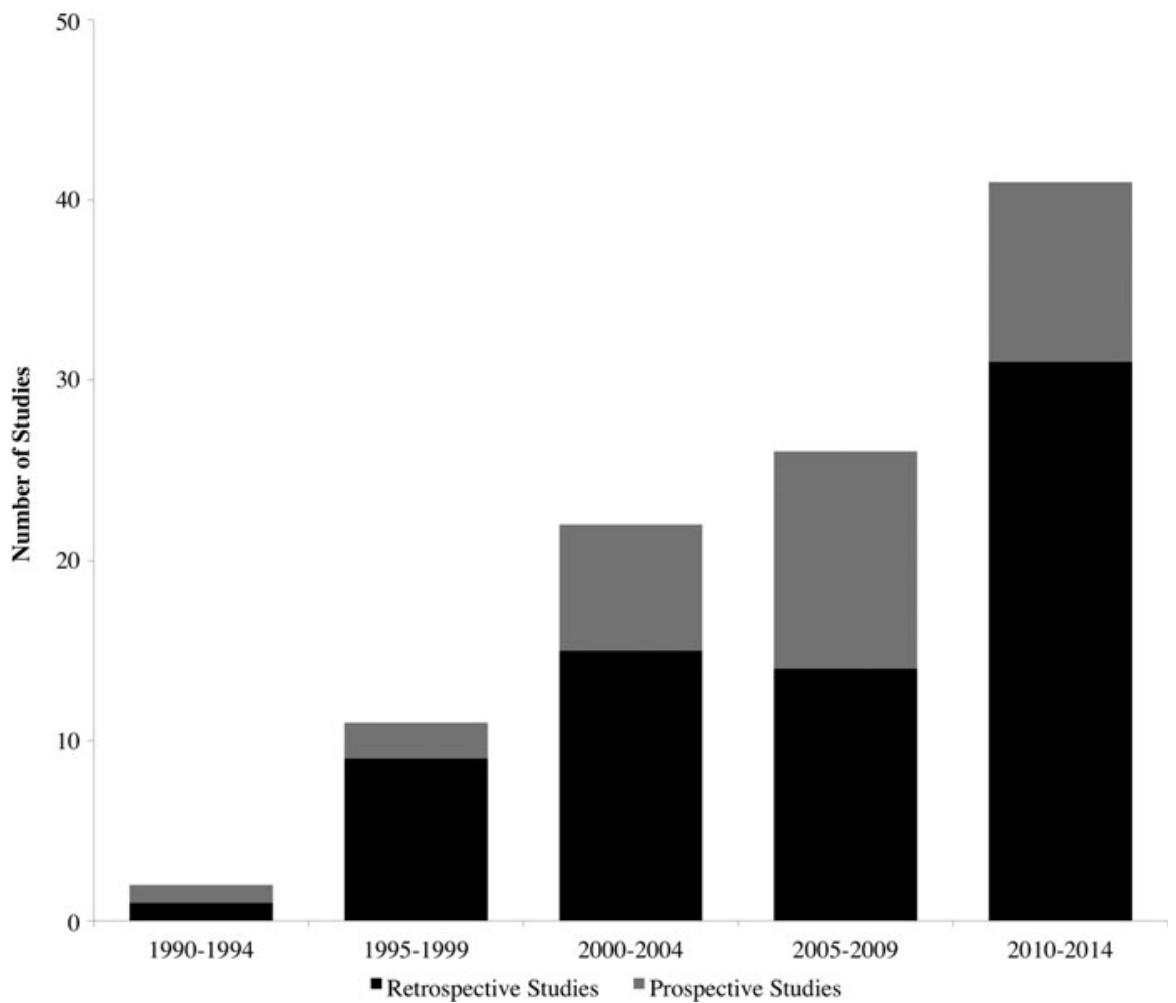

FIG. 3. Stacked area chart illustrating the number of prospective and retrospective studies included, stratified by year of publication. Note that the literature search was conducted in early 2014, so this may be an underestimate of the true number.

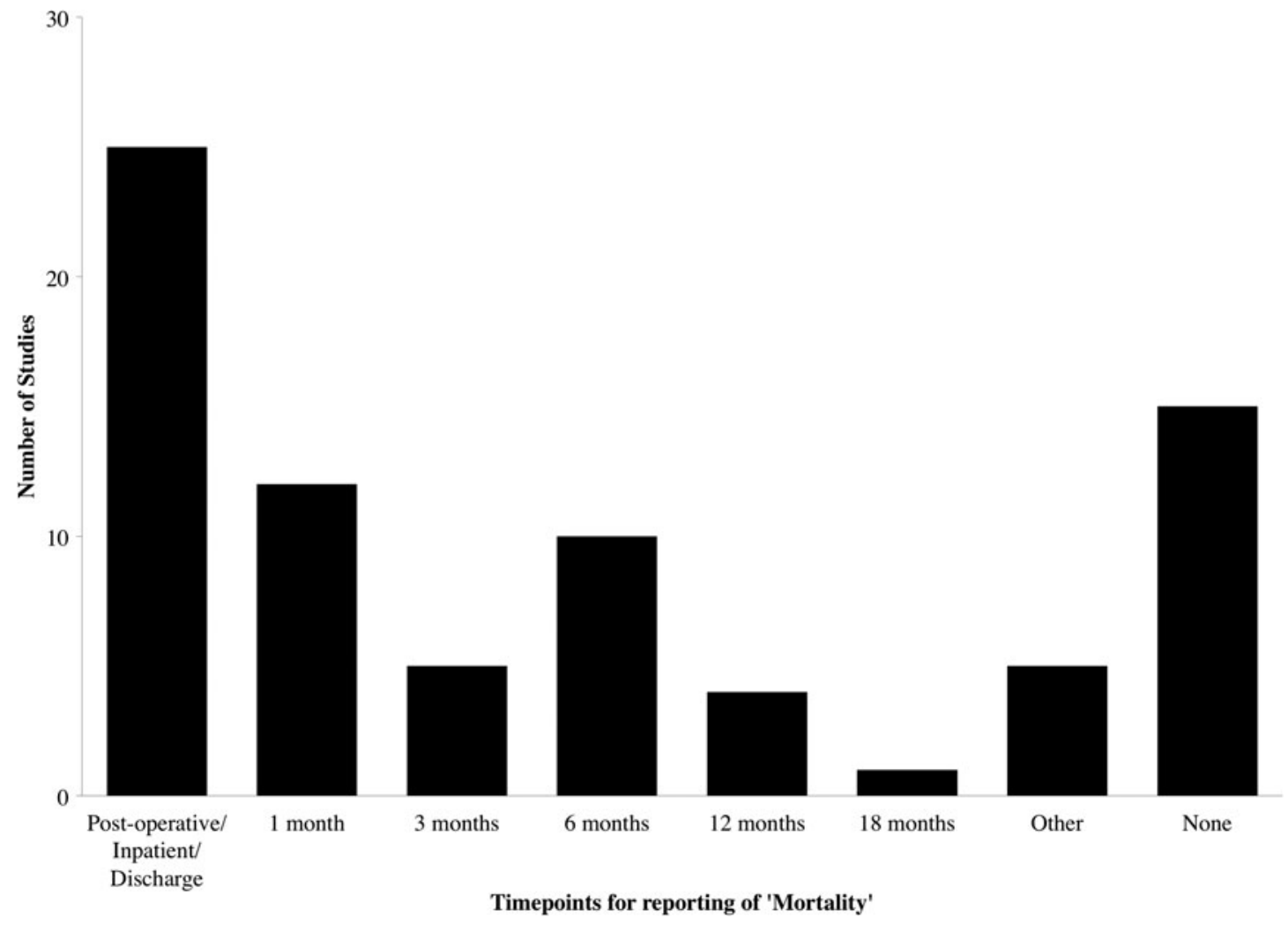

FIG. 4. Time-points of studies reporting mortality. 


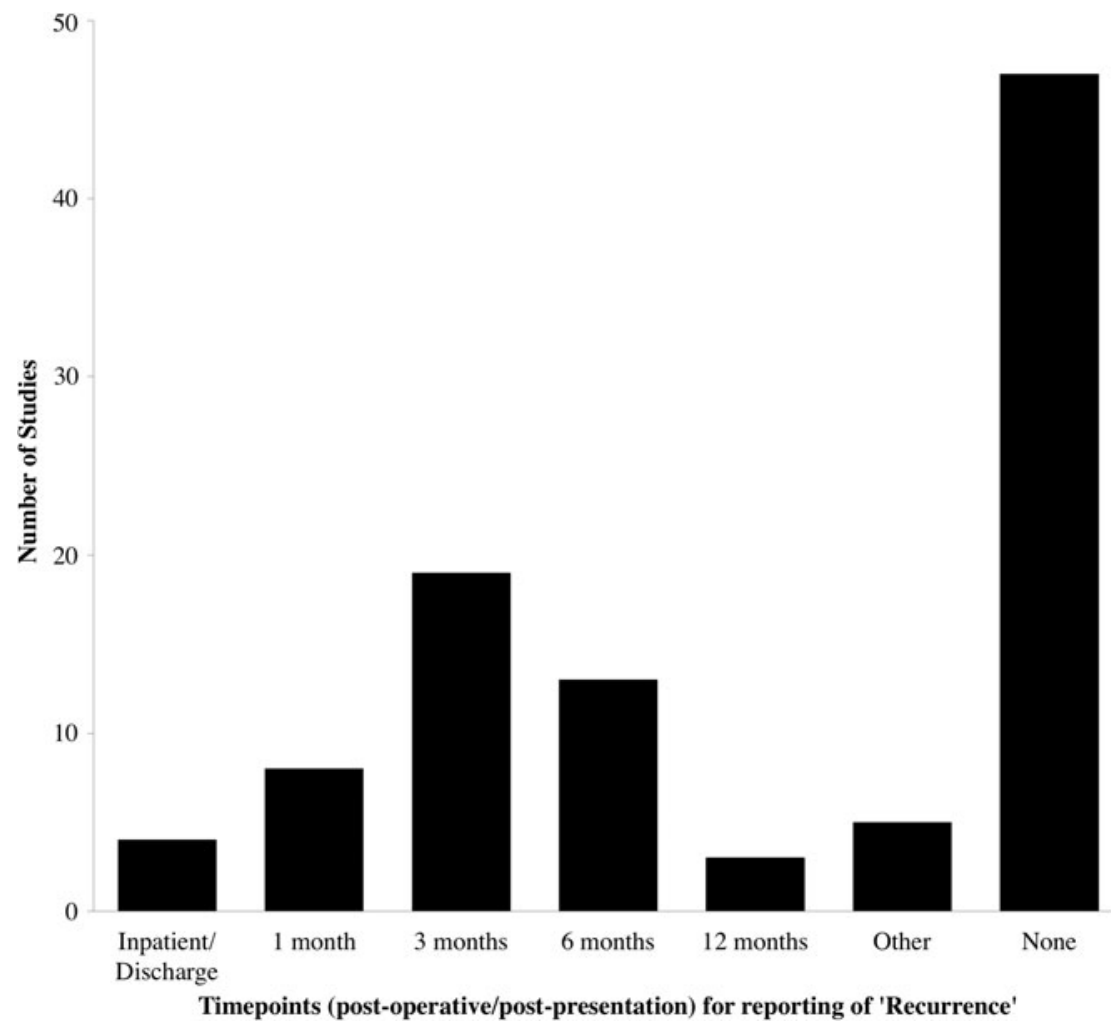

FIG. 5. Time-points for reporting of "recurrence" in 96 chronic subdural hematoma studies.

complications. None of the studies classified the complications according to severity or an established classification system (e.g., the Clavien-Dindo classification). ${ }^{15}$ The time-points were specified in only $16(15.7 \%)$ studies and varied between "post-operative" and 18 months.

\section{Functional outcomes}

Forty-one (40.2\%) studies reported some sort of functional patient outcome, although five $(4.9 \%)$ of these were subjective assessments and eight studies $(7.8 \%)$ reported the Glasgow Coma Scale as an "outcome measure." Some of the common functional assessments included the Glasgow Outcome Scale (GOS; 15 studies, 14.7\%), the Markwalder Scale (14 studies, 13.7\%), and the modified Rankin Scale (mRS; five studies, 4.9\%), Many of the studies measured multiple functional outcomes at multiple time- points, with the most common time-points being at discharge and at 3 and 6 months (Fig. 6).

Approximately one-third of the studies $(31,30.4 \%)$ reported the length of stay; however, the discharge criteria were seldom described and the discharge destination was only described in seven studies $(6.9 \%)$.

\section{Radiological outcomes}

Thirty-nine (38.2\%) studies examined a radiological outcome measure, mostly with post-operative CT scanning. Outcome measures used included thickness of post-operative subdural collection (11 studies, 10.8\%), volume of the post-operative subdural collection (five studies, 4.9\%), midline shift (seven studies, 6.9\%), the presence of intra-cranial air (11 studies, 10.8\%), assessment of drain catheter tip position (seven studies, 6.9\%) and

Table 1. Percentage of Studies Providing Various Definitions for the Term "Recurrence”

\begin{tabular}{lcrc}
\hline & $\begin{array}{c}\text { A: All studies } \\
(\mathrm{n}=96)\end{array}$ & $\begin{array}{c}\text { B: Subgroup of studies looking } \\
\text { at recurrence }(\mathrm{n}=22)\end{array}$ & $\begin{array}{c}\text { C: Subgroup of } \\
\text { RCTs }(\mathrm{n}=12)\end{array}$ \\
\hline No definition & $19.8 \%$ & $4.5 \%$ & $25.0 \%$ \\
Reoperation only & $16.7 \%$ & $9.1 \%$ & $8.3 \%$ \\
Radiological recurrence & $9.4 \%$ & $18.2 \%$ & $8.3 \%$ \\
Radiological recurrence, requiring reoperation & $2.1 \%$ & $4.5 \%$ & $0.0 \%$ \\
Symptomatic or radiological recurrence & $6.3 \%$ & $9.1 \%$ & $8.3 \%$ \\
Symptomatic or radiological recurrence, requiring reoperation & $6.3 \%$ & $4.5 \%$ & $0.0 \%$ \\
Symptomatic recurrence, requiring reoperation & $2.1 \%$ & $0.0 \%$ & $0.0 \%$ \\
Symptomatic and radiological recurrence & $8.3 \%$ & $13.6 \%$ & $16.7 \%$ \\
Symptomatic and radiological recurrence, requiring reoperation & $29.2 \%$ & $36.4 \%$ & $33.3 \%$ \\
\hline
\end{tabular}

Subgroup analyses of studies specifically looking at recurrence $(n=22)$ and RCTs $(n=12)$ show this heterogeneity persists even in the so-called higher quality studies.

RCT, randomized controlled trial. 


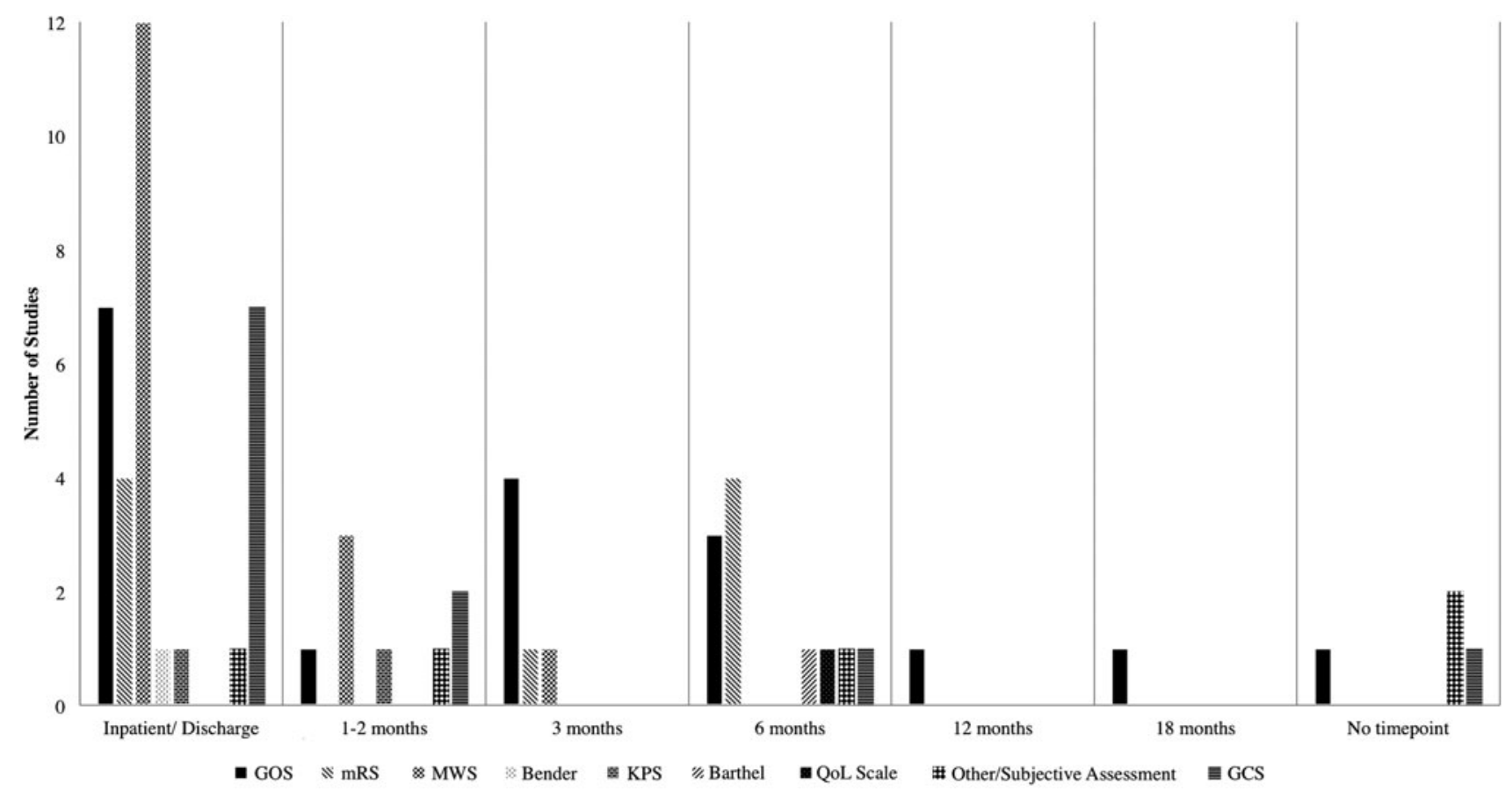

FIG. 6. Functional outcomes and time-points used in the 41 studies that reported functional outcomes.

dichotomous assessments of "resolved," "residual/recurrent" collections (19 studies, 18.6\%). The time-points of these postoperative scans varied from $3 \mathrm{~h}$ post-op up to 6 months. A number of studies followed strict post-operative protocols for scanning (e.g., at 1 day, 1 week, 1 month, 3 months, and 6 months postoperatively) and five studies $(4.9 \%)$ did not specify when postoperative scans were performed.

\section{Discussion}

This systematic review highlights the heterogeneity of outcome measures used in clinical studies assessing treatment efficacy in $\mathrm{CSDH}$. Given that there remains significant variability in management approaches to $\mathrm{CSDH},{ }^{4}$ it builds a strong case for the development of a core outcome set that would homogenize the outcome domains and definitions for future studies. Such a core outcome set would promote the collection of robust outcome data in individual studies, aiding cross-study comparisons and the development of evidence-based treatment algorithms and guidelines for this common neurosurgical condition.

The findings of the present study are not surprising. Such heterogeneity in the measurement of outcome reporting has been established in a number of other specialties, including general surgery, ${ }^{16-19}$ intensive care, ${ }^{20}$ and obstetrics. ${ }^{21}$ Specifically to $\mathrm{CSDH}$, difficulties in amalgamating data from individual studies due to heterogeneous definitions and outcome measures have been identified as limitations in all three large contemporary metaanalyses of $\mathrm{CSDH}^{2,4,7}$ However, the systematic evaluation and quantification of this heterogeneity is a crucial stepping-stone to the informed development of the core outcome set for CSDH.

\section{Outcome domains}

A number of the different outcome domains identified in this study (Table 2), such as mortality and complications are crucial to any disease process, and specifically to surgical conditions, where

thorough evaluation of morbidity and mortality is necessary to evaluate both efficacy and safety. The consensus process will be crucial in identifying a structured way of identifying and classifying morbidity/complications (e.g., the Clavien-Dindo classification), ${ }^{15}$ which are often under-reported in retrospective studies.

Historically, the most important outcome domain in CSDH has been recurrence, as it is a relatively disease-specific outcome measure. This is reflected in the number of studies that reported this outcome $(96 ; 94.1 \%)$ and the number of studies that set out to specifically examine factors that influenced recurrence (22; $21.6 \%$ ). However, as we identified during our recent systematic review of minimally-invasive techniques for $\mathrm{CSDH},{ }^{22}$ the heterogeneity in definitions and time-points of recurrence precludes amalgamation of data and undermines any attempt at meta-analysis.

Recurrence will no doubt remain a cornerstone of the evaluation of treatment options for CSDH, but recent emphasis has shifted towards a more holistic assessment provided by global functional outcome measures such as the GOS and mRS. ${ }^{3,5}$ The GOS was used in $14.7 \%$ of the studies, whereas the mRS was used in $4.9 \%$. These global assessment tools are not specific to CSDH and were originally developed for evaluating functional outcomes in severe TBI $(\mathrm{GOS})^{23}$ and stroke (mRS). ${ }^{24}$ Another issue revolves around

Table 2. Outcome Domains Identified in the Present Study

Outcome domain

Mortality

Complications

Recurrence

Functional outcome

Radiological outcome
Number of studies including outcome domain

$65(63.7 \%)$

$49(48.0 \%)$

$96(94.1 \%)$

$41(40.2 \%)$

$39(38.2 \%)$ 
analysis of these scales; historically, both have been reported as a dichotomized outcome into "good" and "poor," but recent evidence suggests ordinal analysis increases statistical power and should therefore be the preferred option. ${ }^{25}$

In addition to the holistic assessment of the patient, the detailed assessment of surgical and peri-operative complications is crucial in the decision-making process of whether or not to offer CSDH patients surgical intervention. It was noteworthy that only $48.0 \%$ of the studies reported complications and none of them used an established classification system. In addition to surgical complications, it is also important to reinforce the importance of reporting medical complications, especially in the elderly population, which is more likely to suffer adverse effects from conditions like hospital-acquired infections.

The utility of radiological outcomes as a domain remains contentious, especially considering the radiation exposure, cost, and limited utility associated with routine early post-operative CT scans. Certainly, at our institution, routine post-operative scanning is not undertaken and is based on clinical need and there is recent data to suggest that it does not influence post-operative management. ${ }^{26}$

\section{Study limitations}

The limitations of the current study relate to the search criteria. Selecting only English language full-text studies was driven by the observation that definitions of outcomes are often omitted in abstracts/short reports and would be difficult to elicit from nonEnglish language studies. In addition, the date limitation was set to ensure a contemporary cohort of studies measuring relevant out-

\section{CORE OUTCOME SET}

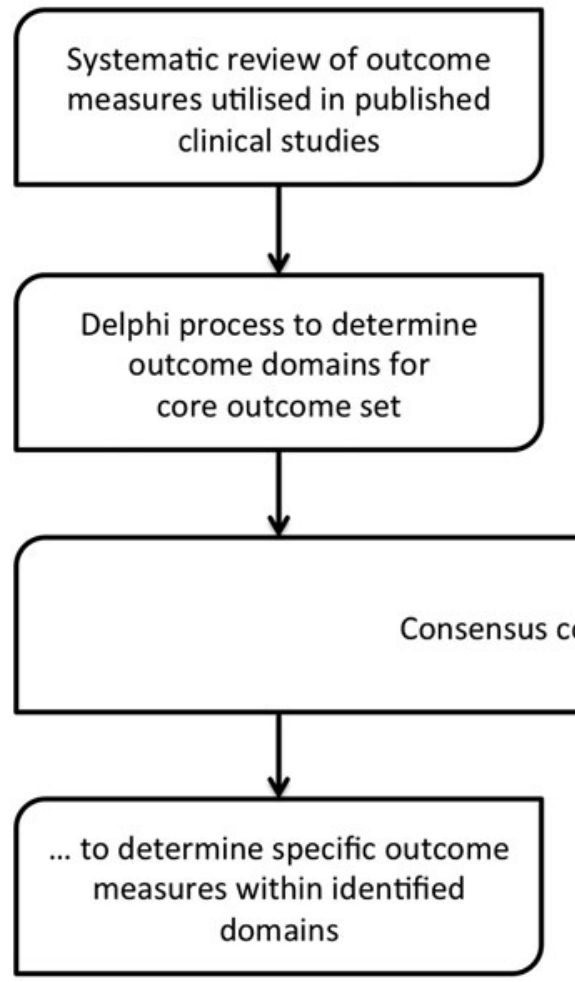

FIG. 7. Aims and overview of the Core Outcomes and Common Data Elements in Chronic Subdural Hematoma (CODE-CSDH) project. comes. Despite these limitations, we feel we have successfully satisfied the aims of the study in providing a strong basis for the consensus process to occur.

\section{Future directions}

As stated in the introduction, this systematic review represents the first step in the process of developing a core outcome set for $\mathrm{CSDH}$. It has illustrated the heterogeneity of outcome measures used in the current CSDH literature and has successfully built a case for the development of a consensus-based core outcome set.

We aim to derive the core outcome set for CSDH via a combination of established methods for developing core outcome sets. ${ }^{14}$ First, we aim to conduct a structured Delphi survey of all relevant stakeholders with clear pre-determined criteria for achieving a majority opinion to determine the outcome domains and confirm/ refine this outcome set via a consensus conference.

In addition to the development of a core outcome set, the CODECSDH project (Fig. 7) also aims to develop a set of common data elements for CSDH. This would standardize the data elements to be collected by future CSDH studies, ensuring homogenous and consistent reporting of demographic data, pre-operative status, and intra-operative details to further facilitate cross-study comparisons and amalgamation of data across studies.

\section{Conclusion}

This systematic review highlights the heterogeneity of outcome reporting in clinical efficacy studies of CSDH. It demonstrates the

\section{COMMON DATA ELEMENTS}

Systematic review of data elements collected in published clinical studies

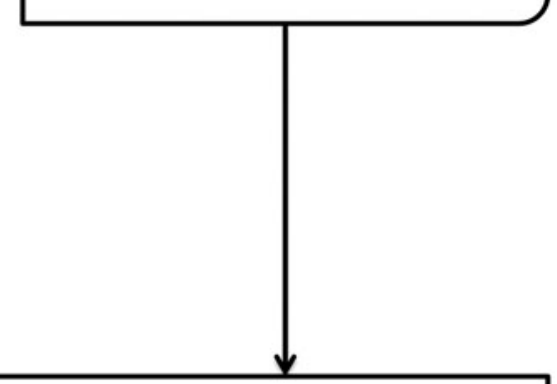

onference ...

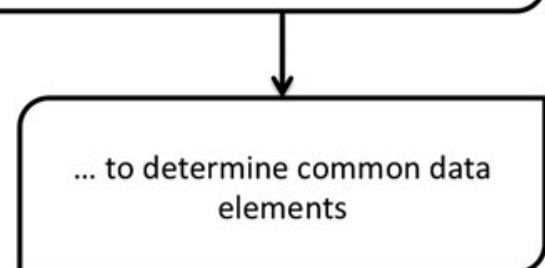


lack of a robust evidence-based approach to the management of this common neurosurgical condition and identifies the need for the development of a robust, patient-centered set of core outcome measures for CSDH as part of the CODE-CSDH project.

\section{Acknowledgments}

We would like to thank Professor Paula Williamson, Professor Jane Blazeby and Miss Elizabeth Gargon from the Core Outcome Measures in Effectiveness Trials (COMET) initiative (www .comet-initiative.org/) for useful discussion and guidance in the course of the CODE-CSDH project.

PJH is supported by a National Institute for Health Research (NIHR) Research Professorship and the NIHR Cambridge Biomedical Research Centre.

\section{Author Disclosure Statement}

No competing financial interests exist.

\section{References}

1. Santarius, T., Kirkpatrick, P.J., Kolias, A.G., and Hutchinson, P.J. (2010). Working toward rational and evidence-based treatment of chronic subdural hematoma. Clin. Neurosurg. 57, 112-122.

2. Ducruet, A.F., Grobelny, B.T., Zacharia, B.E., Hickman, Z.L., DeRosa, P.L., Anderson, K., Sussman, E., Carpenter, A., and Connolly, E.S., Jr. (2012). The surgical management of chronic subdural hematoma. Neurosurg. Rev. 35, 155-169.

3. Kolias, A.G., Chari, A., Santarius, T., and Hutchinson, P.J. (2014). Chronic subdural haematoma: modern management and emerging therapies. Nat. Rev. Neurol. 10, 570-578.

4. Almenawer, S.A., Farrokhyar, F., Hong, C., Alhazzani, W., Manoranjan, B., Yarascavitch, B., Arjmand, P., Baronia, B., Reddy, K., Murty, N., and Singh, S. (2013). Chronic subdural hematoma management: a systematic review and meta-analysis of 34829 patients. Ann. Surg. 259, 449-57.

5. Santarius, T., Kirkpatrick, P.J., Ganesan, D., Chia, H.L., Jalloh, I., Smielewski, P., Richards, H.K., Marcus, H., Parker, R.A., Price, S.J., Kirollos, R.W., Pickard, J.D., and Hutchinson, P.J. (2009). Use of drains versus no drains after burr-hole evacuation of chronic subdural haematoma: a randomised controlled trial. Lancet 374, 1067-1073.

6. Miranda, L.B., Braxton, E., Hobbs, J., and Quigley, M.R. (2011). Chronic subdural hematoma in the elderly: not a benign disease. J. Neurosurg. 114, 72-76.

7. Weigel, R., Schmiedek, P., and Krauss, J.K. (2003). Outcome of contemporary surgery for chronic subdural haematoma: evidence based review. J. Neurol. Neurosurg. Psychiatry 74, 937-943.

8. Williamson, P.R., Altman, D.G., Blazeby, J.M., Clarke, M., Devane, D., Gargon, E., and Tugwell, P. (2012). Developing core outcome sets for clinical trials: issues to consider. Trials 13, 132.

9. Williamson, P.R., Altman, D.G., Blazeby, J.M., Clarke, M., and Gargon, E. (2011). The COMET (Core Outcome Measures in Effectiveness Trials) Initiative. Trials 12, A70.

10. Kirkham, J.J., Boers, M., Tugwell, P., Clarke, M., and Williamson, P.R. (2013). Outcome measures in rheumatoid arthritis randomised trials over the last 50 years. Trials 14, 324 .

11. Saver, J.L., Warach, S., Janis, S., Odenkirchen, J., Becker, K., Benavente, O., Broderick, J., Dromerick, A.W., Duncan, P., Elkind, M.S., Johnston, K., Kidwell, C.S., Meschia, J.F., and Schwamm, L. (2012). Standardizing the structure of stroke clinical and epidemiologic research data: the National Institute of Neurological Disorders and Stroke (NINDS) Stroke Common Data Element (CDE) project. Stroke 43, 967-973.

12. Maas, A.I., Harrison-Felix, C.L., Menon, D., Adelson, P.D., Balkin, T., Bullock, R., Engel, D.C., Gordon, W., Langlois-Orman, J., Lew, H.L., Robertson, C., Temkin, N., Valadka, A., Verfaellie, M., Wainwright, M., Wright, D.W., and Schwab, K. (2011). Standardizing data collection in traumatic brain injury. J. Neurotrauma 28, 177-187.

13. Budhdeo, S., Chari, A., Harrison, O., and Blazeby, J. (2014). Patientcentred healthcare outcome measures: towards a unified architecture J. R. Soc. Med. 107, 300-302.

14. Gargon, E., Gurung, B., Medley, N., Altman, D.G., Blazeby, J.M., Clarke, M., and Williamson, P.R. (2014). Choosing important health outcomes for comparative effectiveness research: a systematic review. PLoS One 9, e99111.

15. Dindo, D., Demartines, N., and Clavien, P.A. (2004). Classification of surgical complications: a new proposal with evaluation in a cohort of 6336 patients and results of a survey. Ann. Surg. 240, 205-213.

16. Blencowe, N.S., McNair, A.G., Davis, C.R., Brookes, S.T., and Blazeby, J.M. (2012). Standards of outcome reporting in surgical oncology: a case study in esophageal cancer. Ann. Surg. Oncol. 19, 4012-4018.

17. Blencowe, N.S., Strong, S., McNair, A.G., Brookes, S.T., Crosby, T., Griffin, S.M., and Blazeby, J.M. (2012). Reporting of short-term clinical outcomes after esophagectomy: a systematic review. Ann. Surg. 255, 658-666.

18. Bruce, J., Russell, E.M., Mollison, J., and Krukowski, Z.H. (2001). The measurement and monitoring of surgical adverse events. Health Technol. Assess. 5, 1-194.

19. Bruce, J., Krukowski, Z.H., Al-Khairy, G., Russell, E.M., and Park, K.G. (2001). Systematic review of the definition and measurement of anastomotic leak after gastrointestinal surgery. Brit. J. Surg. 88, $1157-1168$

20. Hayes, J.A., Black, N.A., Jenkinson, C., Young, J.D., Rowan, K.M., Daly, K. and Ridley, S. (2000). Outcome measures for adult critical care: a systematic review. Health technol. assess. 4, 1-111.

21. Kritchevsky, S.B., Braun, B.I., Gross, P.A., Newcomb, C.S., Kelleher, C.A., and Simmons, B.P. (1999). Definition and adjustment of Cesarean section rates and assessments of hospital performance. Int. J. Qual. Health Care 11, 283-291.

22. Chari, A., Kolias, A.G., Bond, S., Santarius, T., and Hutchinson, P.J. (2014). Twist-drill craniostomy with hollow screws for evacuation of chronic subdural haematoma: a systematic review. J. Neurosurg. 121, 176-183.

23. Jennett, B. and Bond, M. (1975). Assessment of outcome after severe brain damage. Lancet 1, 480-484.

24. van Swieten, J.C., Koudstaal, P.J., Visser, M.C., Schouten, H.J., and van Gijn, J. (1988). Interobserver agreement for the assessment of handicap in stroke patients. Stroke 19, 604-607.

25. Roozenbeek, B., Lingsma, H.F., Perel, P., Edwards, P., Roberts, I., Murray, G.D., Maas, A.I., and Steyerberg, E.W. (2011). The added value of ordinal analysis in clinical trials: an example in traumatic brain injury. Crit. Care 15, R127.

26. Ng, H.Y., Ng, W.H., and King, N.K. (2014). Value of routine early post-operative computed tomography in determining short-term functional outcome after drainage of chronic subdural hematoma: an evaluation of residual volume. Surg. Neurol. Int. 5, 136.

Address correspondence to:

Angelos G. Kolias, MRCS Department of Clinical Neurosciences Addenbrooke's Hospital and University of Cambridge Hills Road, Cambridge, CB2 OQQ United Kingdom E-mail: angeloskolias@gmail.com 